(Aus dem physiologischen Institut der Universität Marburg.)

\title{
Beschreibung einiger Apparate für das physiologische Praktikum.
}

I.

Modification des Riva-Rocei'schen und Gärtner'schen Blutdruckmessers. Vereinfachter Tonograph.

Von

F. Schenck.

Die neuen Vorschriften über die ärztlichen Prüfungen für das Deutsche Reich haben eine wichtige Neuerung im physiologischen Unterricht gebracht. Für den Studirenden der Medicin ist die Theilnahme an einem physiologischen Praktikum vorgeschrieben worden. Während bisher an den meisten deutschen Universitäten, soviel mir bekannt, nur wenige Studirende sich an den physiologischen Uebungen betheiligten, die Mehrzahl sich auf die Theilnahme an physiologisch-chemischen Cursen beschränkte, sollen jetzt sämmtliche Studirende in der Experimentalphysiologie praktjsch unterwiesen werden.

Bei der Durchführung dieser Vorschrift wird sich für die physiologischen Institute die Nothwendigkeit ergeben, eine grössere Zahl von Apparaten eigens für Unterrichtszwecke anzuschaffen.

Für die Construction solcher Apparate werden zwei Gesichtspunkte maassgebend sein müssen:

1. Die Apparate müssen möglichst einfach und übersichtlich gebaut sein; alle didaktisch-wertlosen Complicationen sind wegzulassen; dabei muss aber doch den Anforderungen wissenschaftlicher Genauigkeit genügt sein.

2. Die Apparate müssen billig sein, so dass ihre Anschaffung in grösserer Zahl, sowie auch der Ersatz, der voraussichtlich in Folge des starken Verbrauchs oft nothwendig sein wird, leicht ohne erhebliche Mittel möglich ist. 
Von diesen Gesichtspunkten habe ich mich bei der Einrichtung der Curse im hiesigen Institute leiten lassen, und ich bin dabei zu einigen Neuconstructionen gelangt, über die ich wohl einige Mittheilungen machen darf, weil sie auch für Andere Interesse haben könnten.

Nicht unerwähnt darf ich lassen, dass ich bei der Herstellung der Apparate von dem Mechaniker des hiesigen physiologischen Instituts, Herrn M. Rinck, dem ich manchen nützlichen Wink verdanke, in wirksamer Weise unterstützt wurde.

Ich beginne mit der Beschreibung einiger Apparate für die Untersuchung des Blutdrucks.

Für die Blutdruckmessung beim Menschen kommen gegenwärtig in der ärztlichen Praxis hauptsächlich das Sphygmomanometer von Riva-Rocci und das Tonometer von Gärtner in Betracht. Mit diesen beiden wird der Mediciner im physiologischen Praktikum bekannt zu machen $\operatorname{sein}^{1}$ ).

Von den oben erwähnten Gesichtspunkten aus habe ich folgende Theile modificirt:

\section{Die Absperrvorrichtung.}

Als Absperrvorrichtung dient bei beiden Apparaten bekanntlich ein pneumatischer Ring, der beim Riva-Rocei um den Oberarm, beim Gärtner um einen Finger gelegt und aufgebläht wird; der aufgeblähte Ring übt einen Druck auf die Weichtheile der Glieder aus, und es wird bestimmt, wie gross der Druck im pneumatischen Ring ist, der dem arteriellen Blutdruck gerade das Gleichgewicht hält.

Die Absperrvorrichtung ist bei beiden Apparaten mit Mängeln behaftet. Die wesentlichsten Theile der Absperrvorrichtung bestehen aus Gummi und werden daher leicht undicht, wenn der Apparat einige Zeit in Verwendung gewesen ist. Besonders den grossen

1) Die Bekanntschaft mit diesen Apparaten darf ich bei den Lesern des Archivs voraussetzen, so dass eine Beschreibung und Kritik derselben hier unnöthig ist. Ich verweise auch auf die neuesten Arbeiten von H. v. Recklinghausen (Arch. f. exp. Path. u. Pharm. Bd. 46) und Max Neu (Experim. u. klin. Untersuchungen mit Gärtner's Tonometer. Heidelberg 1902), in denen die Methoden einer kritischen Prüfung unterworfen sind und frühere Literatur eingehend berücksichtigt ist. 
Absperrschlauch des Riva-Rocei dicht zu bekommen und zu erhalten, ist sehwierig.

Da übrigens Gummi allein zu nachgiebig ist, um die erforderlichen Drucke auf die Glieder zu Stande kommen zu lassen, so sind beide Absperrvorrichtungen noch mit unnachgiebigen Hüllen versehen. Beim Riva-Rocei besteht die Hülle aus Seidenzeug, das aber bei öfterem Gebrauche leicht verschleisst. Beim Gärtner ist die Absperrvorrichtung durch einen starrwandigen Metallring eingeschlossen; dieser Ring passt natürlich nur für einen Finger von bestimmter Grösse; um an verschieden grossen Fingern Bestimmungen nach Gärtner's Methode machen zu können, muss man einen ganzen Satz von Absperrringen zur Verfügung haben; auch können Fehler dadurch entstehen, dass der starre Ring sich dem Gliede nicht genügend anschmiegt ${ }^{1}$ ).

Für die Neuconstruction kam es also darauf an, die Absperrvorrichtung aus einem Material zu construiren, das haltbarer ist als Gummi, das sich den Gliedern leicht anschmiegt, dabei aber doch so unnachgiebig ist, dass es einer besonderen unnachgiebigen Hülle nicht bedarf.

Ausserdem musste die Absperrvorrichtung des modificirten Gärtner von variabler Weite sein, damit ein und dieselbe Absperrvorrichtung für Finger versehiedener Grösse verwendbar war.

Als vorzügliches Material für die Absperrvorrichtung habe ich einen Stoff gefunden, der aus besonders präparirtem gummirten feinen Leder besteht. Die Firma Martin Wallach Nachfolger in Cassel hat auf meinen Wunsch Absperrvorrichtungen aus Gummileder berstellen lassen, welche sich als recht brauchbar erwiesen haben. Das Gummileder ist nach Angabe des Lieferanten ich selbst habe darüber noch keine Erfahrung - haltbarer und dabei billiger als Gummi.

Die Absperrvorrichtung hat folgende Form erhalten: Sie besteht aus einem an beiden Enden offenen Stück Gummilederschlauches, das für die beiden Apparate verschieden gross ist. Für den modificirten Riva-Rocei ist der platt hingelegte Lederschlauch $10 \mathrm{~cm}$ breit $^{2}$ ),

1) Auf diese Uebelstände ist auch schon von v. Recklinghausen und $\mathrm{N}$ eu hingewiesen worden (a. a. O.)

2) Der Schlauch ist breiter als der früher dem Riva-Rocei beigegebene. Dass von der Breite des Schlauches die Genauigkeit der Messung abhängt, ist von v. Recklinghausen (a. a. O.) gezeigt worden. Dieser empfiehlt sogar, den 
$45 \mathrm{~cm}$ lang, für den modificirten $\mathrm{Gärtner} 3 \mathrm{~cm}$ breit, $14 \mathrm{~cm}$ lang. Ungefähr in der Mitte des Schlauches hat seine Wand ein kleines, kreisrundes Loch, durch das ein beiderseits offenes Messingröhrchen durchgesteckt ist. Das in das Schlauchlumen hineinragende Ende des Messingröhrchens ist hier zu einem Metallplättchen verbreitert, und dieses Plättchen wird gegen die Schlauchwand angepresst durch eine Schraubenmutter, welche von aussen her um das Röhrchen gegen die Schlauchwand zu gedreht wird. In dieser Weise ist das Röhrchen luftdicht in die Schlauchwand einzusetzen. Das äussere Ende des Röhrchens wird durch Gummischlauch mit dem Manometer und der Druckvorrichtung (siehe unten) verbunden.

Die beiden Enden des Lederschlauches werden durch eine Schraubenklemme gesteckt und durch Anziehen der Klemmschraube zugepresst. So ist der pneumatische Ring hergestellt, und die Weite des Ringes kann dem jeweiligen Bedürfnisse entsprechend variirt werden dadurch, dass man die Schlauchenden bald mehr, bald weniger weit durch die Schraubenklemme hindurchzieht. In analoger Weise wurde ja bisher schon der Riva-Rocci'sche Schlauchring variirt; in derselben Weise kann auch der modificirte Gärtnersche Schlauchring den Bedürfnissen angepasst werden. Die Weite der Schlauchringe wird so bemessen, dass sie den Gliedern gerade leicht anliegen, ohne eng zu schliessen oder zu drücken.

\section{Das Manometer.}

Da mir die Anschaffung der für die Curszwecke nothwendigen grösseren Zahl von Quecksilber- oder Metallmanometern zu theuer war, so habe ich ein Manometer aus ganz einfachen Theilen zusammengesetzt, das besonderes Interesse auch für den ärztlichen Praktiker haben dürfte, weil es ein leicht transportables Quecksilbermanometer darstellt. Es besteht aus folgenden Theilen:

Ein niedriges, breites Fläschchen $(1 \mathrm{~cm}$ hoch, von $4 \mathrm{~cm}$ Durchmesser) mit niedrigem Halse ist etwa zur Hälfte mit Quecksilber

Schlauch noch breiter zu machen, aber da die ganz breiten Schläuche etwas unbequem zu handhaben sind, und da sich unser neue Schlauch aus dem feinen Leder auch leichter an die Glieder anschmiegt, als die früher verwandten Schläuche, so dürfte die Breite unseres Schlauches genügen, insbesondere auch, weil es bei den klinischen Untersuchungen hauptsächlich ankommt auf vergleichende Messungen, die an ein und derselben Persun unter verschiedenen Umständen vorzunehmen sind. 
gefüllt; es wird gewöhnlich mit Kork- oder Gummipfropf verschlossen gehalten und kann, so geschlossen, auch bequem transportirt werden. Zur Herrichtung des Manometers wird in den Hals des Fläschchens ein doppelt durchbohrter Gummipfropf fest eingesetzt. Dieser trägt in der einen Durchbohrung eine etwa $25 \mathrm{~cm}$ lange dickwandige Glasröhre von $1 \mathrm{~mm}$ Lumen, deren unteres Ende in das Quecksilber taucht, und die als Steigröhre für das Manometer dient. Die Höhe, bis zu der das Quecksilber steigt, kann leicht abgelesen werden an einer an der Glasröhre zu befestigenden Scala. Die Scala befindet sich auf einem Pappstreifen, der unten einen für das Fläschchen passenden Ausschnitt hat; die Scala wird so gestellt, dass ihr Nullpunkt in der Höhe des Quecksilberniveaus im Fläschchen steht. Die Ablesung des Quecksilberniveaus in der Steigröhre ist natürlich nur oberhalb des Gummipfropfens, d. i. von etwa $30 \mathrm{~mm} \mathrm{Hg-Säule}$ an, möglich, aber das genügt, weil die niedrigeren Druckwerthe für unsere Bestimmungen nicht in Betracht kommen. Da das Lumen der Steigröhre sehr eng ist, das Fläschchen dagegen sehr breit, so wird das Quecksilberniveau im Fläschchen nur unmerklich sinken, wenn das Quecksilber in der Steigröhre steigt. Die Veränderung des Nullpunkts kommt also für die Messung nicht in Betracht, weil sie zu unerheblich ist. Dasselbe gilt ja übrigens auch für das dem Riva-Rocci bisher beigegebene Quecksilbermanometer. Auf das obere Ende des Steigrohrs wird noch ein mit kleinem Loch versehenes Gummihütchen aufgesetzt, um Herausspritzen des Quecksilbers bei unvorsichtiger Drucksteigerung zu verhindern.

In die zweite Durchbohrung des Gummipfropfens wird der eine Schenkel eines T-Rohres so eingesetzt, dass das in das Fläschchen hineinragende Ende dieses Schenkels noch nicht in das Quecksilber eintaucht. Der zweite Schenkel des T-Rohres ist durch einen Gummischlauch in Verbindung mit dem Metallröhrchen der Absperrvorrichtung; der dritte wird verbunden mit der Druckvorrichtung.

\section{Die Druckvorrichtung.}

Für den Riva-Rocei besteht die bisherige Druckvorrichtung in einem Gummigebläse, mit dem Luft in die Absperrvorrichtung hineingetrieben wird, so lange, bis der Puls in der Arteria radialis ausbleibt. Dieselbe Druckvorrichtung lässt sich auch für meine 
Modification des Riva-Rocci verwenden. Nur wird in die Leitung von dem Gummigebläse zu dem Manometer noch ein zweites T-Rohr einzuschalten sein, an dessen dritten, noch freien Schenkel ein kurzes, durch Quetschbahn 'zu schliessendes Gummischlauchstück angesetzt wird. Durch Oeffnen des Quetschhahns ist nach der Druckmessung die eingetriebene Luft wieder zu entfernen.

Auch für den modificirten Gärtner kann die bisherige Druckvorrichtung beibehalten werden. Der Druck wird hier bekanntlich gesteigert dụrch Compression eines Gummiballons zwischen einer Schraubenpresse. Nach Anlegen der Absperrvorrichtung an den Finger wird aus diesem durch Ueberstreifen eines kleinen Gummiringes das Blut verdrängt, dann durch Zuschrauben der Presse der Druck so weit erhöht, dass er sicher grösser ist, als der arterielle Blutdruck, dass also die Gefässe comprimirt werden und das Blut vom Finger abgesperrt gehalten wird. Dann wird der Gummiring vom Finger abgenommen und nun durch Zurückschrauben der Schraubenpresse der Druck so weit gesenkt, bis eben das Blut in den blassen Finger wieder einströmt.

Um indess auch bei der Druckvorrichtung die wenig haltbaren Gummiballons resp. Gummigebläse zu vermeiden, habe ich auch hierfür Ballons aus Gummileder herstellen lassen, für den Gärtner einen solchen von $100 \mathrm{ccm}$, für den Riva-Rocei von $500 \mathrm{ccm}$ Inhalt in aufgeblähtem Zustande. In diese Ballons, die durch Ansatzstück und Schlauchverbindung mit dem Manometer und den Absperrvorrichtungen verbunden werden, wird der Druck wie bei dem alten Gärtner'schen Apparat durch Zuschrauben einer Schraubenpresse von passender Grösse gesteigert. Um dann aber den Lederballon vor Beginn des Versuches schon mit Luft zu füllen, was für die Erzielung eines grossen Druckes von Vortheil ist, wird auch hier in die Leitung vom Ballon zum Manometer ein zweites T-Rohr eingeschaltet, dessen freier Schenkel wieder mit Gummiansatzstück zu versehen und mit Quetschhahn zu schliessen ist. Vor Beginn des Versuches bläst man von diesem Gummistück aus den Lederballon schon auf, verschliesst dann das Ansatzstück und kann nun die erhebliche Drucksteigerung leicht vornehmen.

Uebrigens habe ich noch beim modificirten $G$ är tner'sehen Apparat gerade für die Zwecke des physiologischen Unterrichts eine viel einfachere und leicht $\mathrm{zu}$ beschaffende andere Druckvorrichtung verwendet. Dieselbe besteht einfach aus einem $30 \mathrm{~cm}$ langen Gummi- 
schlauch von etwa $8 \mathrm{~mm}$ lichter Weite und $1,5 \mathrm{~mm}$ Wandstärke, dessen eines Ende an den für die Druckvorrichtung bestimmten Schenkel des Manometer-T-Rohres angesetzt wird. Der Druck wird hier in folgender Weise gesteigert. Man klemmt das freie Ende des Schlauches zunächst mit Daumen und Zejgefinger einer Hand zu, setzt dann vor die zugeklemmte Stelle gegen das Manometer zu Daumen und Zeigefinger der anderen Hand und comprimirt; dann werden vor diese comprimirte Stelle wieder die Finger der ersten Hand gesetzt und so fort. Auf diese Weise vertreibt man die Luft aus dem Schlauche in das Manometer und in die Absperrvorrichtung, und kann dadurch leicht einen Druck von der gewünschten Höhe erzeugen. Hat man den Druck erreicht, so klemmt man den Schlauch mit einer Klemmpincette oder einem Quetschhahn zu. Diese Klemmvorrichtung wird, wenn der Druck gesenkt werden soll, dann vorsichtig ein wenig geöffnet. Dum Anfänger wird es dabei aber leicht vorkommen, dass er die Klemmvorrichtung zu schnell und zu weit öffnet, so dass der Druck sehr schnell und beträchtlich sinkt. Um dies bequem zu vermeiden, drückt man den Schlauch mit Daumen und Zeigefinger der linken Hand $\mathrm{zu}$ an einer Stelle etwa $1-2 \mathrm{~cm}$ hinter der Stelle, wo die Klemmvorrichtung liegt, und löst dann erst mit der rechten Hand die Klemmvorrichtung. Es wird dann nur wenig Luft aus dem Manometer in das kleine abgegrenzte Schlauchstück ubertreten können und der Druck nur um ein Geringes sinken. Man wiederholt diese Manipulation mehrmals hinter einander und kann so den Druck bequem absatzweise bis zu der Grösse sinken lassen, wo die Röthung des Fingers beginnt. Diese Druckvorrichtung ist so einfach und so leicht zu ersetzen, auch bei einiger Uebung so bequem zu handhaben, dass ich sie auch für die in der ärztlichen Praxis verwendeten Apparate empfehlen kann.

Die hier beschriebenen modificirten Riva-Rocei'schen und Gärtner'schen Apparate werden von der Firma Martin Wallach Nachfolger in Cassel in den Handel gebracht.

Für Blutdruckmessungen in vivisectorischen Versuchen, die wohl am besten an Kaninchen anzustellen sind, stehen uns die gebräuchlichsten Quecksilber- und elastischen Manometer zur Verfügung. Am leichtesten auszuführen sind die Versuche mit den neueren Tonographen, z. B. dem Hürthle'schen, dem v. Frey'schen u. A. Aber man wird diese werthvollen und difficilen Apparate nicht gerne 
428 F. Schenck: Beschreibung einiger Apparate f. d. physiol. Praktikum.

dem Anfänger in die Hand geben. Ich habe mich daher bemüht, auch einen vereinfachten Tonographen speciell für Unterrichtszwecke zu construiren. Der Apparat fiel so einfach aus, dass es geboten erschien, durch eine sorgfältige Prüfung festzustellen, ob die Leistungsfähigkeit des Apparates auf Kosten der Einfachheit nicht sehr beschränkt ist. Herr Dr. Ishihara hat die Prüfung vorgenommen; er wird in dem folgenden Aufsatze über die Ergebnisse der Prüfung Bericht erstatten und bei dieser Gelegenheit den Apparat genauer beschreiben.

Ueber eine Reihe weiterer speciell für das physiologische Praktikum gebauter Apparate soll in späteren Mittheilungen berichtet werden.

Nachtrag: Während der Drucklegung des Vorstehenden hatte ich Gelegenheit, beim modificirten Riva-Rocci ein kleines Metallgebläse auszuprobiren, das nach Art der Pumpen für die Pneumatiks der Fahrräder gebaut war. Dieses Gebläse hat sich als brauchbar erwiesen, und dürfte wegen seiner Haltbarkeit und bequemen Handbabung nicht nur dem Gummigebläse, sondern auch dem Gummilederballon vorzuziehen sein, zumal da man sich das Ventil des Metallgebläses leicht selbst repariren kann. 